\title{
Intermédialité et transfigurations dans le cycle du Survenant
}

\author{
David Décarie \\ Université de Moncton
}

L'une des forces de l'écriture de Germaine Guèvremont est la cohérence et la richesse des réseaux d'images et de figures de style ${ }^{1}$ qui forment la trame ses romans. Loin d'être de simples ornements, les figures forment une architecture complexe et constituent un enjeu central de l'écriture. Le Cycle du Survenant ${ }^{2}$ de Guèvremont ne se borne toutefois pas au roman :

\footnotetext{
1 J'emprunte à Laurent Jenny sa définition de la figure : « La figure est une forme typique de relation entre des éléments discursifs.»(2003, Les figures de rhétorique, Méthodes et problèmes, Genève, Département de français moderne <http://www.unige.ch/lettres/framo/enseignements/methodes/frhetorique/> 2 Selon Besson (2004, p. 22), qui étudie les cycles et les séries, « le retour d'au moins un personnage ou d'au moins un nom propre $\mathrm{p}[\mathrm{eu}] \mathrm{t}$ être considéré comme la conditionsine qua non de constitution d'un ensemble ».
} 
intermédiale et plurigénérique, son œuvre s'ouvre par un recueil de nouvelles (En pleine terre, 1942), se continue dans deux romans (Le Survenant, 1945; Marie-Didace, 1947) avant d'être approfondie et poursuivie sous forme de radioroman (CBF 1952-1955, CKVL 1962-1965) puis de téléroman ${ }^{3}$ (CBFT 1954-1960). Dans cet article, je tenterai de mieux comprendre l'effet du changement de genre littéraire sur les figures de style en suivant l'adaptation des figures des trois premiers chapitres du roman Le Survenant aux médias radiophonique et télévisuel. L'étude des figures du premier chapitre me permettra de cerner quelques-uns des défis que pose l'adaptation des figures. Nous verrons qu'il est impossible d'analyser leurs transformations sans réfléchir aux spécificités génériques de leur emploi, notamment au niveau narratif. En analysant les figures du second chapitre du roman, j'étudierai le rôle joué par celles-ci dans l'expression de la subjectivité des personnages et je montrerai les changements provoqués par la transition des genres à ce niveau. En abordant les figures du troisième chapitre, enfin, je montrerai que Guèvremont a procédé à une réécriture complète de certaines scènes afin de réussir à transposer certaines figures importantes.

\section{Genres et figures}

L'analyse du premier chapitre du roman Le Survenant permet de souligner quelques-unes des difficultés qui se posent à

\footnotetext{
${ }^{3}$ La matière d'un troisième roman que prévoyait écrire l'auteure a finalement donné lieu aux deux dernières séries télévisées (Marie-Didace et Le Survenant, CBFT 1958-1960) dans lesquelles Guèvremont a introduit une nouvelle génération de personnages. Le Survenant, vieilli, marqué par la guerre, revient dans la dernière série pour sceller le destin des Beauchemin. La fin du cycle n'existe ainsi que sous forme télévisée.
} 
Guèvremont dans la transposition des figures de son roman. Celles-ci commencent dès l'incipit, avec la curieuse majuscule attribuée au nom commun « survenant » :

Un soir d'automne, au Chenal du Moine, comme les Beauchemin s'apprêtaient à souper, des coups à la porte les firent redresser. C'était un étranger de bonne taille, jeune d'âge, paqueton au dos, qui demandait à manger.

- Approche de la table. Approche sans gêne, Survenant, lui cria le père Didace ${ }^{4}$. $(S$, p. 83$)$

L'antonomase (" prendre un nom propre pour un nom commun ou un nom commun pour un nom propre », Dupriez, p.58) contribue au caractère hiératique et poétique de l'incipit et, par elle, l'attribution du nom s'apparente à un baptême, ce que viendra confirmer le passage du personnage sous l'eau baptismale de la pompe des Beauchemin. Guèvremont, dans la suite du roman, renouvelle d'ailleurs le choc initial de l'antonomase en mettant en relation le nom commun «survenant» et les noms propres du héros («Survenant» ou «Venant»): «Survenant, survenant, remarqua Venant, vous avez toujours ce mot-là à la bouche. Dites-moi une fois pour toutes ce que vous entendez par là » $(S$, p. 114). La popularité du personnage a quelque peu émoussé l'audace de l'antonomase, qui nous semble aujourd'hui aller de soi. Guèvremont elle-même n'y arriva pourtant pas du premier jet : dans la première version du chapitre parue dans Gant $d u$ ciel $^{4}$, le mot « survenant » reste un nom commun.

4 « L'arrivée de Venant » et « L'abandon », Gants du ciel, no 2, décembre 1943, p. 21-32 (première version des chapitres I et XVII du Survenant). 
Quel est le sort de l'antonomase dans le radioroman et le téléroman? Apparaissant dans un dialogue, la figure se prête bien, à première vue, à la transposition générique : la réplique du père Didace, comme la plupart des dialogues du roman, est reprise intégralement dans les adaptations et reproduite telle quelle dans les scénarios. Et pourtant, comme la majorité des figures romanesques, l'antonomase n'est pas directement transposable dans les nouveaux médias. Le lecteur aura bien sûr deviné que l'antonomase repose sur un code graphique étranger au radioroman et au téléroman : la majuscule, en effet, ne s'entend pas dans la langue parlée. La mise en relation avec les genres «parlés » fait toutefois apparaître le paradoxe de son emploi dans le roman : l'appartenance de l'antonomase au discours du père Didace n'est qu'une illusion d'optique, car la figure est étrangère à la diégèse et ne peut qu'être attribuée à la narration. Dès cette première figure, nous sommes ainsi confrontés à l'enjeu important du statut narratif des figures.

La perte d'une figure, dans les adaptations, peut souvent être compensée. La principale stratégie de l'auteure, dans l'adaptation des figures, consiste à contourner les obstacles. Elle réussit ainsi à intégrer la tension nom commun / nom propre en reprenant du roman des figures voisines qui sont plus facilement transposables, soit l'aphérèse (" On retranche une syllabe ou une lettre au commencement d'un mot.», Dupriez, p. 60) et la paronomase ( Rapprochement de mots dont le son est à peu près semblable, mais dont le sens est différent. », Dupriez, p. 332). On en trouve un exemple dans l'extrait suivant $t^{5}$ :

5 Germaine Guèvremont, Le Survenant, Radioroman (CBF, 31 août 1953 - 6 mai 1955; repris à CKVL, septembre 1962 - juin 1965), Bibliothèque et 


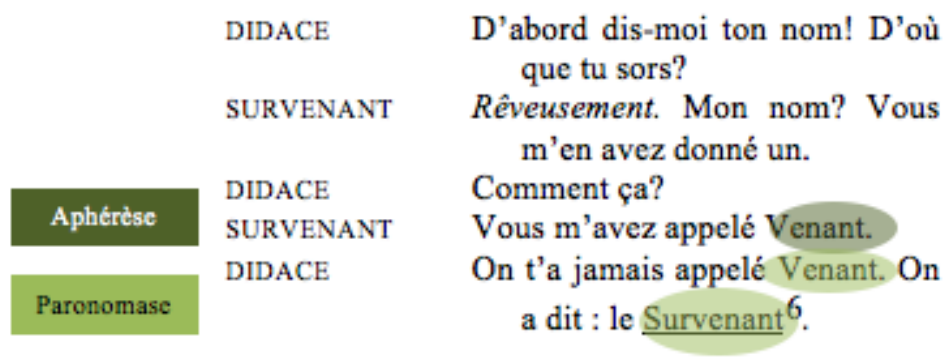

Le changement de genre rend manifeste une évidence : les genres n'offrent pas tous les mêmes possibilités aux figures, ils ne constituent pas un milieu neutre. Les genres radiophonique et télévisuel modifient en profondeur les figures romanesques et leur organisation. Les différences génériques (présence ou absence d'une narration) ou médiatiques (le roman est lu, le radioroman entendu, et le téléroman vu et entendu) modulent l'utilisation des figures et font que celles-ci «passent» ou «ne passent pas». Une partie du travail de l'auteure, dans l'adaptation et la réécriture de ses romans, consiste justement à essayer de "faire passer» la matière romanesque à travers les différences génériques. Dans le cas des figures, ce défi est souvent important, car tout n'est pas transposable. Chez Guèvremont comme chez de nombreux romanciers, les figures abondent dans les pauses narratives que sont les descriptions. Or celles-ci disparaissent presque complètement dans les adaptations: privée d'une narration extradiégétique --réservée, on le verra, à la transition des

archives Canada, Fonds Germaine Guèvremont, LMS 0260, 2004-03, série 1, no 5 , 28 sept. 1962, p. 6-7. Désormais, les références à cet ouvrage seront indiquées par le sigle $R R S$, suivi du numéro de l'épisode, de la date de la mise en ondes, du numéro de page du scénario, et placées entre parenthèses dans le texte. 
épisodes--, l'auteure ne sait littéralement plus où les mettre. Comme les lecteurs du roman, les auditeurs du radioroman ne voient pas la chemise du Survenant, et pourtant, malgré sa pertinence, la description suivante disparaît :

D'un simple signe de la tête, sans même un mot de gratitude, l'étranger accepta. Il dit seulement :

- Je vas toujours commencer par nettoyer le cochon.

Après avoir jeté son baluchon dans l'encoignure, il enleva sa chemise de laine à carreaux rouge vif et vert à laquelle manquaient un bouton près de l'encolure et un autre non loin de la ceinture. $(S$, p. 84$)$

Dans le téléroman, bien sûr, les descriptions à caractère visuel peuvent être directement montrées (ou plutôt transposées dans des codes sémiotiques visuels) ${ }^{6}$.

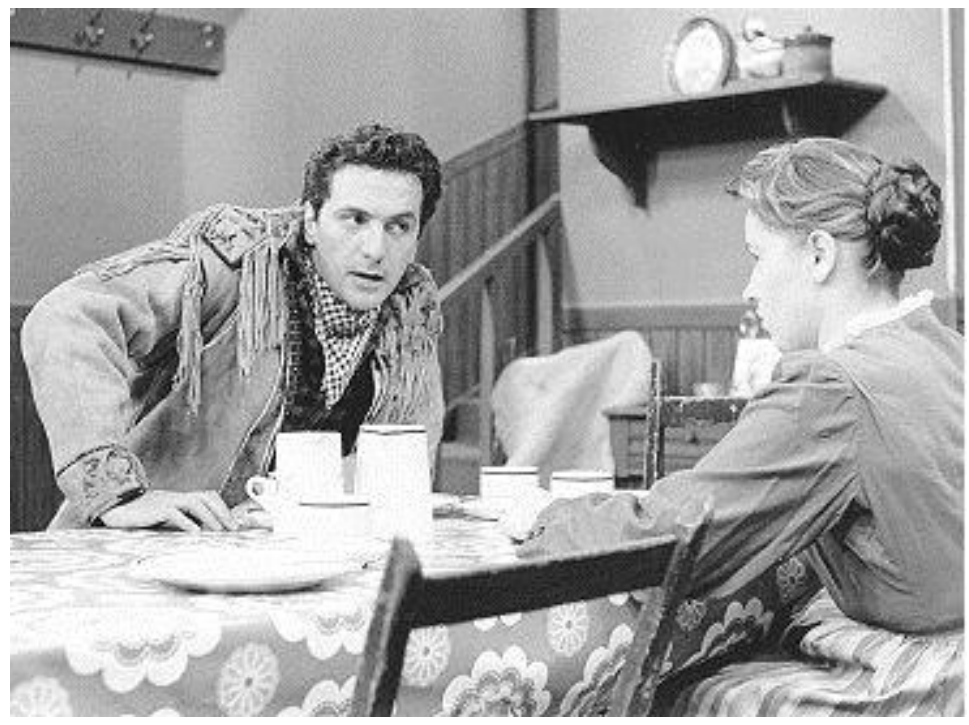

6 Tous les médias imposent toutefois des contraintes. La télévision, à ses débuts, n'existe ainsi qu'en noir et blanc et n'aurait pu rendre les « carreaux rouge vif et verts » de la chemise du Survenant. 
La découverte d'une veste indienne par une accessoiriste de Radio-Canada devait toutefois radicalement transformer le Grand-dieu-des-ondes. L'adaptation visuelle la plus fidèle n'aurait toutefois pu rendre le travail du style, les homéotéleutes (« On place à la fin des phrases ou des membres de phrase des mots de même finale », Bernard Dupriez, p. 232) «brodés » par Guèvremont dans la veste de son personnage :

Après avoir jeté son baluchon dans l'encoignure, il

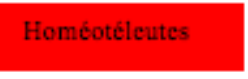
enleva sa chemise de laine à carreaux rouge vif et vert à laquelle manquaient un bouton près de l'encolure et un autre non loin de la ceinture, ( $S$, p. 84$)$

La transformation radiophonique du passage illustre toutefois l'une des transformations possibles des figures; cellesci peuvent être prolongées, ou filées :

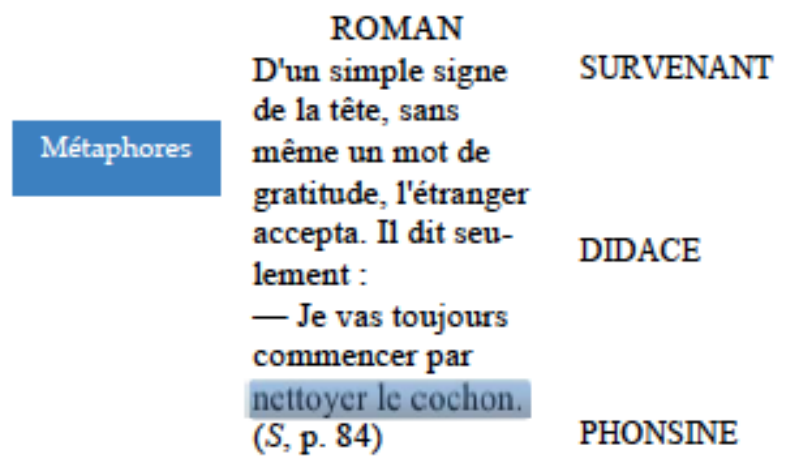

RADIOROMAN
Dans ce moment-là,
j'vas toujours com-
mencer par nettoyer le
cochon. Rire.
La pompe est là. Le
savon du pays puis
l'essuie-main à côté.
T'as beau! Bruit de
pompe.
(Mi-voix) Le cochon?
On dirait qu'il se
connaît. (RRS, n' 1,24
sept. 1962, p. 6$)$


La métaphore devient alors un enjeu des échanges verbaux des personnages : Phonsine, par son réemploi ironique, lui donne un sens nouveau.

Analysant le dialogue romanesque à l'aune de l'analyse conversationnelle, Véronique Traverso remarque le «passage de la multicanalité de la communication authentique à la «monocanalité » du texte » (1999, p. 105). Comme le souligne Alain Rabatel, les informations narratives, dans le roman, passent par un canal unique :

les informations arrivent au lecteur directement, par le biais du narrateur, ou médiatement, par celui du personnage. Cette répartition serait simple si chacune de ces instances possédait un canal spécifique par où transiterait l'information. Ce n'est pas le cas. (Rabatel, 1998, p. 13)

Le passage du roman au radioroman et au téléroman est justement marqué par la multiplication des canaux d'informations: dans ces genres, en effet, le narrateur et les différents personnages possèdent tous une voix distincte. $\mathrm{Au}$ niveau des figures, une partie du travail d'adaptation consiste donc à répartir celles-ci sur les différents canaux. Il s'agit d'un travail ardu, notamment parce que la voix de la narration extradiégétique est considérablement réduite dans les adaptations. Dans le radioroman, le narrateur extradiégétique se réduit le plus souvent au rôle d'un présentateur chargé d'introduire et de conclure l'épisode ${ }^{7}$ et ce dernier disparaît à peu près totalement dans le téléroman.

Le statut narratif des figures est essentiel, car certaines figures, prises en charge par la narration (je propose le terme de

\footnotetext{
${ }^{7} \mathrm{Au}$ niveau générique, la disparition du narrateur permet au radioroman de se singulariser, d'être autre chose qu'un feuilleton lu.
} 
«figures de la narration »), sont extérieures au récit - ou extradiégétiques - tandis que d'autres, intradiégétiques, sont employées par les personnages (je parlerai de «figures du personnage »). Le passage du baptême symbolique du Survenant, dans l'incipit du roman, donne une bonne idée des défis que pose la transposition des figures de la narration :

De ses mains extraordinairement vivantes l'étranger s'y baigna le visage, s'inonda le cou, aspergea sa chevelure, tandis que les regards s'acharnaient à suivre le moindre de ses mouvements. On eût dit qu'il apportait une vertu nouvelle à un geste pourtant familier à tous. (S, p. 84)

Dans cet extrait, on peut repérer au moins trois hyperboles et une comparaison antithétique. Ces figures sont prises en charge par une narration extradiégétique à focalisation zéro (narration qui se distingue par une absence de point de vue). Le changement de genre provoque une réorganisation complète de celles-ci :

\begin{tabular}{|c|c|c|c|c|}
\hline $\begin{array}{l}\text { Pléonasme } \\
\text { Hyperbole } 1\end{array}$ & $\begin{array}{c}\text { Hyperboles } \\
2,3\end{array}$ & & Hyperbole 1 & $\begin{array}{c}\text { Hyperbole } 2 \\
\text { Métaphore }\end{array}$ \\
\hline \multicolumn{2}{|c|}{$\begin{array}{c}\text { ROMAN } \\
\text { Figures de la narration } \\
\text { (extradiégétiques) }\end{array}$} & & \multicolumn{2}{|c|}{$\begin{array}{l}\text { RADIOROMAN } \\
\text { Figures du personnage (in- } \\
\text { tradiégétiques) }\end{array}$} \\
\hline \multirow{7}{*}{\multicolumn{2}{|c|}{$\begin{array}{l}\text { De ses mains extraordinai- } \\
\text { rement vivantes létranger } \\
\text { s'y baigna le visage, } \\
\text { s'inonda le cou, aspergea sa } \\
\text { chevelure, tandis que les } \\
\text { regards s lacharnaient à sui- } \\
\text { vre le moindre de ses mou- } \\
\text { vements. On eût dit qu'il } \\
\text { apportait une vertu nouvelle } \\
\text { à un geste pourtant familier } \\
\text { à tous. }(S, \text { p. } 84)\end{array}$}} & & \multirow{7}{*}{\multicolumn{2}{|c|}{$\begin{array}{l}\text { Bas. C'est-ti comique de le voir } \\
\text { se laver? } \\
\text { J'ai jamais vu un homme pren- } \\
\text { dre tant de temps. Il s'en va pas } \\
\text { aux noces. } \\
\text { Ça fait du bien de se laver... de } \\
\text { se baigner le visage... de } \\
\text { s'inonder le cou... la chevelu- } \\
\text { re... L'eau manque pas par ici } \\
\text { d'après ce que je peux voir. } \\
\left.\text { (RRS, } \mathrm{n}^{\circ} 1,24 \text { sept } 1962, \text { p. } 6\right)\end{array}$}} \\
\hline & & PHONSINE & & \\
\hline & & & & \\
\hline & & SURV & & \\
\hline & & & & \\
\hline & & & & \\
\hline & & & & \\
\hline \multicolumn{2}{|c|}{$\begin{array}{l}\text { Comparaison } \\
\text { Antithèse }\end{array}$} & & Hyper & Litote \\
\hline
\end{tabular}


Hormis les rares interventions du narrateur, il n'est qu'un destin possible pour les figures de la narration dans les adaptations: elles doivent devenir intradiégétiques en se faisant «adopter» par un personnage. Le Survenant, dans le radioroman, verbalise ainsi l'hyperbole 2 du roman et prend en charge la description de l'action. Pour diverses raisons, toutes les figures de la narration ne peuvent êtres transformées en figures d'un personnage. La comparaison antithétique faite par la narration ( «On eût dit qu'il apportait une vertu nouvelle à un geste pourtant familier à tous.» S, p. 84) n'est ainsi pas transposable, car elle provient d'un point de vue extérieur et porte sur l'ensemble des protagonistes dont elle traduit la "pensée», voire les impressions. Toutefois, les répliques de Beau-Blanc et Phonsine rendent, de façon très différente il est vrai, l'étrangeté du « geste familier » fait par le Survenant.

Danielle Aubry a noté la position centrale du personnage dans les téléromans québécois :

[C]ontrairement aux feuilletons états-uniens, les personnages ne sont pas dominés par une action qui les manipule comme autant de pantins, mais forment le cœur même du téléroman, son «lieu commun », sa force empathique, émotive, qui crée d'emblée un lien puissant avec le téléspectateur[.] (Aubry, p. 160)

L'observation vaut également pour les radioromans. Le fait que le personnage y devient le principal «filtre » des figures romanesques contribue d'ailleurs à son importance. Hors du personnage, point de salut pour les figures. À la radio comme à la télévision, le personnage obéit à un pacte réaliste : il possède des caractéristiques physiques, mentales et sociales de même qu'un registre langagier auxquels devront s'adapter les figures. Le pléonasme disparaît ainsi dans le radioroman parce qu'il enfreindrait ce pacte réaliste : aucun des protagonistes ne peut 
en effet s'extasier sur les "mains extraordinairement vivantes » du Survenant parce que cela ne s'accorderait ni au registre langagier ni aux conventions sociales qui règlent leurs échanges verbaux.

Ces extraits soulignent toutefois l'effort fait par Guèvremont pour garder une proximité rhétorique avec l'épisode romanesque, notamment en forgeant de nouvelles hyperboles pour remplacer celles qui n'ont pu être transposées (la figure supplémentaire qui apparaît, la litote, est elle-même voisine de l'hyperbole). Il faut toutefois noter le passage du registre du sacré vers celui du quotidien. Le ton hiératique et poétique installé par la narration extradiégétique fait place à l'anecdotique, et même, avec la tirade du personnage de BeauBlanc, au « comique ».

Le travail d'adaptation, chez Guèvremont, est facilité par l'importance qu'elle accorde aux personnages dans ses romans. L'auteure s'intéresse certes à l'espace socio-historique que constitue le Chenal du Moine, mais son centre d'intérêt réside avant tout dans les personnages, et tout particulièrement, on le verra, dans leur interrelation. Reflétant cette préférence, les figures de la narration sont, dans le roman, nombreuses à graviter autour des personnages qu'elles permettent d'approfondir et de nuancer.

Le soin avec lequel Guèvremont transpose ses figures dans les adaptations montre également que celles-ci jouent un rôle essentiel dans le récit. L'un des meilleurs exemples de leur importance est sans doute l'emploi que fait la romancière de l'antithèse dans les premiers chapitres du Survenant: cette dernière lui permet, avec une grande économie de moyens, de 
décrire les personnages, d'établir leurs relations et de souligner les tensions qui existent entre eux :

\section{Antithèse à 4 termes (A, B, C, D)}

À l'encontre [A] des hommes qui [B] buvaient par lampées [C] dans des tasses de faïence grossière d'un blanc crayeux, cru, et parfois aussi dans des bols qu'ils voulaient [D] servis à la rasade, quelle qu'en fût la grandeur, [A'] la jeune femme [B'] aimait boire à petites gorgées, [C'] dans une tasse de fantaisie [D'] qu'elle n'emplissait jamais jusqu'au bord. Après qu'il en eut avalé suffisamment, 1'étranger consentit à dire :

- C'est un bon thé, mais c'est pas encore un vrai thé de chanquier. Parlez-moi d'un thé assez fort qu'il porte la hache, sans misère! ( $S$, p. 86)

Personnification

Hyperbole

L'antithèse est ici formée de quatre séries d'oppositions :

\begin{tabular}{|l|l|}
\hline$[\mathrm{A}]$ des hommes & {$\left[\mathrm{A}^{\prime}\right]$ la jeune femme } \\
\hline$[\mathrm{B}]$ buvaient par lampées & $\begin{array}{l}{[\mathrm{B} \text { '] aimait boire à peti- }} \\
\text { tes gorgées }\end{array}$ \\
\hline $\begin{array}{l}{[\mathrm{C}] \text { dans des tasses de }} \\
\text { faïence grossière d'un } \\
\text { blanc crayeux, cru, et } \\
\text { parfois aussi dans des bols }\end{array}$ & $\begin{array}{l}\text { [C'] dans une tasse de } \\
\text { fantaisie }\end{array}$ \\
\hline$[\mathrm{D}]$ servis à la rasade & $\begin{array}{l}\text { [D'] qu'elle n'emplissait } \\
\text { jamais jusqu'au bord }\end{array}$ \\
\hline
\end{tabular}


On peut considérer que la personnification hyperbolique du thé prend également place dans l'antithèse, car le thé " porteur de hache » s'oppose (et même menace) la fragile tasse de Phonsine, toujours à un doigt de se casser. Notons qu'une antithèse supplémentaire précède cet extrait et oppose l'appétit des hommes à celui de Phonsine qui «pignoch[e] dans son assiette ».

On l'a vu, les figures romanesques, loin d'apparaître $e x$ nihilo, sont en relation avec plusieurs éléments constitutifs ou «catégories» du roman (avec la narration, la temporalité, l'espace, mais également avec les personnages). D'un point de vue textuel, le personnage est un ensemble de mots: «Le personnage est représenté, pris en charge et désigné sur la scène du texte par un signifiant discontinu, un ensemble dispersé de marques que l'on pourrait appeler son "étiquette" » (Hamon, p. 142). Les figures constituent l'une des "marques » de l'étiquette du personnage ${ }^{8}$ qu'elles contribuent à distinguer et à approfondir. Les antithèses, par exemple, reviennent avec une remarquable constance dans l' «étiquette» d'Alphonsine. Cette figure traduit fort bien l'aliénation de la seule femme de la « maison du père ${ }^{9}$ » dans l'idéologie paternaliste.

Faute de place peut-être (les tapuscrits des scénarios ont en moyenne huit pages), l'antithèse disparaît du premier épisode du radioroman. Il est toutefois rare, dans le travail d'adaptation, qu'une figure disparaisse sans laisser de traces. Le manque d'appétit de Phonsine, l'un des éléments de l'antithèse, est ainsi repris et, pour exprimer celui-ci, Guèvremont prête à

\footnotetext{
${ }^{8}$ Les figures peuvent toutefois appartenir à plus d'une catégorie à la fois.

9 Patricia Smart, Écrire dans la maison du père : l'émergence du féminin dans la tradition littéraire du Québec, Montréal, Québec-Amérique, 1988.
} 
Phonsine une comparaison hyperbolique qui appartient à la narration du roman Marie-Didace ${ }^{10}$ : «J'ai pas plus faim que la rivière a soif » $\left(R R S, \mathrm{n}^{\circ} 1,24\right.$ sept 1962, p. 7). De plus, l'auteure forge une nouvelle antithèse qui vient en quelque sorte remplacer celle du roman : « [Didace à Phonsine :] Fais nous de l'ordinaire. Puis fais-nous-en en masse. De la soupe. Pas une soupe clairette qu'on boit dans un bol comme à midi. De la soupe épaisse. (RRS, no 1, 24 sept 1962, p. 63) ${ }^{11}$ » L'antithèse est cependant beaucoup moins riche.

Dans le téléroman, toutefois, Guèvremont transpose fidèlement l'antithèse du roman en reprenant, dans l'ordre mais de façon fragmentée, tous ses termes (sauf l'opposition «hommes/ jeune femme» qui est implicite). Dès sa première émission, l'auteure profite pleinement des possibilités du nouveau média, car l'antithèse devient «intermédiale ». Le premier terme de l'antithèse, se trouvant dans la didascalie, devient à l'écran purement visuel : "Phonsine dispose 3 grosses tasses pour les hommes puis une petite tasse de fantaisie pour $e l l e^{12}$. » La qualité des tasses, plus difficile à rendre à l'écran, est transposée par une opposition de taille. L'antithèse se poursuit dans une réplique du père Didace à Phonsine : «Verses-en! Aie pas peur! Tu sais que j'aime ça... à la rasade... quand ça déborde! » (TRS, no 1, 2 nov. 1954, p. 19; je souligne). Notons la

10 Marie-Didace, édition critique par Yvan G. Lepage, Montréal, Presses de l'Université de Montréal, coll. "Bibliothèque du Nouveau Monde », [1947] 1996, p. 102.

11 Cette antithèse sera par la suite reprise dans le téléroman.

12 Germaine Guèvremont, Le Survenant, Téléroman (CBFT, 30 novembre 1954 au 9 juillet 1957), Bibliothèque et archives Canada, Fonds Germaine Guèvremont, LMS 0260, 2004-03, série 2, no 1, 2 novembre 1954, p. 9. Désormais, les références à cet ouvrage seront indiquées par le sigle $T R S$, suivi du numéro de l'épisode, de la date de la mise en ondes, du numéro de page du scénario, et placées entre parenthèses dans le texte. 
réapparition du terme exact du roman : « à la rasade ». Explicite dans le roman, l'opposition avec Phonsine devient implicite. La fragmentation de l'antithèse permet de plus à Guèvremont d'être plus précise: Phonsine ne s'oppose pas tant à Amable qu'à Didace, qui est associé à l'hyperbole «quand ça déborde $^{13}$ ». L'antithèse se poursuit ensuite au plan visuel :

\section{Antithèse à 2 termes (A, B)}

Phonsine sert tout le monde. Arrivée au point de se servir, elle n'en [A] verse qu'un peu au fond de sa tasse. [...] Le Survenant observe Phonsine qui boit [B] à petites gorgées, puis Beau-Blanc qui [A'] $a$ versé du thé dans sa soucoupe et qui la boit [B'] à grandes lampées[.] (TRS, $\mathrm{n}^{\circ} 1,2$ nov. 1954, p. 20)

Notons que la figure de la narration devient une figure de personnage, car le Survenant, par son regard, la prend en charge. L'antithèse se poursuit enfin dans les tirades des personnages :

$\begin{array}{ll}\text { BEAU-BLANC } & \begin{array}{l}\text { Y'est pas fort, le yable! Va } \\ \text { ben tomber sans connais- } \\ \text { sance. (Petit rire) [...] }\end{array} \\ \text { SURVENANT } & \begin{array}{l}\text { Parlez moi d'un thé qui por- } \\ \text { te la hache sans misère! } \\ \left.\text { (TRS, } \mathrm{n}^{\circ} 1,2 \text { nov. } 1954, \mathrm{p} .20\right)\end{array}\end{array}$

\section{Personnification 1 \\ Hyperbole 1}

Personnification 2

Hyperbole 2

13 L'hyperbole permet aussi, de préciser le sens de l'expression « à la rasade » pour un public qui n'écoute pas nécessairement la télévision avec un dictionnaire sur les genoux. 
L'appartenance de la personnification du thé à l'antithèse, implicite dans le roman, est explicitée et enrichie dans le téléroman par l'ajout d'une seconde personnification dans la bouche de Beau-Blanc. Le thé, dès lors, décrit non seulement l'opposition entre Phonsine et les hommes, mais également l'opposition entre Amable et le Survenant, car Amable, malade, tombera littéralement sans connaissance à la fin du repas. La figure complexe qu'est l'antithèse survit non seulement au changement de genre littéraire, mais se développe et trouve ici un nouveau dynamisme.

Une seconde antithèse importante apparaît dans le premier chapitre et oppose le vieux Didace aux jeunes de la tablée (antithèse elle-même liée à un doublet métaphorecomparaison) :

\section{Antithèse à 4 termes (A, B, C, D)}

Métaphore

Comparaison

Amable, [A] hâtivement, s'en taillait [B] une tranche de deux bons doigts d'épaisseur, [C] sans s'inquiéter de ne pas déchirer la mie. Chacun de la tablée que la faim travaillait l'imita. Le [D'] vieux les observait à la dérobée, l'un après l'autre. Personne, cependant, ne semblait voir l'ombre de mépris qui, petit à petit, comme une brume d'automne, envahissait les traits de son visage austère. Quand vint son tour, lui, Didace, fils de Didace, qui avait le respect du pain, de sa main gauche prit [A'] doucement près de lui la miche rebondie, l'appuya contre sa poitrine deminue encore moite des sueurs d'une longue journée de labour, et, de la main droite, ayant raclé son couteau sur le bord de l'assiette jusqu'à ce que la lame brillât de propreté, [C'] tendrement il se découpa [B'] un quignon de la grosseur du poing. (S, p. 85) 
Soulignons au passage la force expressive de la métaphore chez Guèvremont qui procède ici de ce que l'on pourrait appeler son caractère "transcatégoriel»: les figures mettent en effet en relation les catégories du personnage, de l'espace et de la temporalité. Observons de plus que la comparaison "comme une brume d'automne» opère la «bifurcation ${ }^{14}$ » du sens faible du mot ombre («soupçon, trace. Elle n'avait pas l'ombre d'un remords.), employé littéralement, à son sens le plus fort, («Zone sombre créée par un corps opaque qui intercepte les rayons d'une source lumineuse; obscurité, absence de lumière (surtout celle du soleil) dans une telle zone), pleinement métaphorique. Il est fort difficile de transposer de telles figures hors de la narration extradiégétique. Les métaphores nécessitent un langage spécialisé pouvant difficilement être mis dans la bouche des personnages. Au niveau des figures, les métaphores sont les grandes perdantes de la transposition, mais on verra que l'auteure n'hésite pas à faire des détours considérables pour tenter de les transposer en son et en image. L'antithèse aurait cependant assez facilement pu être intégrée dans le dialogue des personnages, mais Guèvremont abandonne cette figure, peut-être parce que l'auteure recentre l'action du radioroman autour de Phonsine.

Dans le téléroman, toutefois, Guèvremont intègre de façon visuelle l'antithèse du pain, au moyen d'une didascalie :

Il [Amable] prend la miche et commence à tailler le pain tout croche. Le père Didace suit ses mouvements avec une révolte muette. Il prend le pain à son tour, fait une croix avec son couteau,

\footnotetext{
14 Soit le fait qu'un mot ou qu'une série de mots appartienne à plusieurs lexiques métaphoriques à la fois, voir: Roger M. White, The Structure of Metaphor, Cambridge, Blackwell Publishers, 1996, p. 105.
} 
l'appuie sur sa poitrine et le taille droit, avec tranquillité, d'un geste fort et rond. (TRS, no 1,2 nov. 1954, p. 20)

L'antithèse est ainsi recentrée sur l'opposition entre Amable et Didace. Dans une scène antérieure, alors que Didace, entré dans la maison, dispute Phonsine à propos du pain, Guèvremont ajoute une réplique qui étend l'antithèse à Didace et Phonsine en établissant clairement leur différence de statut hiérarchique $^{15}$ :

[Didace à Phonsine:] Combien d'fois qu'faudra t'dire que le pain se coupe à table? Entame le jamais avant. (Pause) C'est à moi Didace Beauchemin, à entamer le pain... pas à toi, Phonsine Ladouceur! (TRS, nº 1, 2 nov. 1954, p. 20)

\section{Figures et subjectivité}

L'analyse du second chapitre du roman et de ses adaptations permet de saisir le rôle joué par les figures dans l'expression de la subjectivité des personnages et de constater les changements importants provoqués par la transition des genres. Patricia Smart a souligné l'importance de l'interrelation des personnages dans les romans de Guèvremont: «La préoccupation de l'identité se déplace vers celle des relations entre les êtres, l'unité fait place à la multitude [...] [L]e "personnage principal" reste [...] entouré de mystère, et n'a d'autre fonction dans l'intrigue que son effet sur les autres personnages» (1988, p. 142). Ces interrelations complexifient l'«étiquette » des personnages chez Guèvremont : celle-ci n'est pas fixe mais varie selon les divers points de vue présentés. Les qualités et les défauts du Survenant font, par exemple, l'objet d'une véritable spéculation de la part de plusieurs

\footnotetext{
15 L'autorité patriarcale écrasante du père Didace, implicite dans le roman, devient explicite dans les adaptations.
} 
des habitants du Chenal du Moine. Un autre procédé récurrent de la romancière consiste à faire d'une pierre deux coups en présentant un personnage A par le biais d'un personnage B. C'est ainsi que dans le second chapitre, dans une longue analepse qui revient sur les heures précédant l'arrivée du Survenant, Guèvremont, par l'emploi de la focalisation interne, centre son récit sur Didace et c'est par son regard que le lecteur verra Phonsine. Subtil et efficace, le procédé permet d'approfondir simultanément ces deux personnages tout en explorant leur relation conflictuelle.

Malgré son importance, le chapitre II disparaît presque totalement des adaptations. Celui-ci est constitué de quatre parties, soit: [A] Didace arrête de labourer la terre pour contempler sa propriété puis [B] il discute avec Pierre-Côme Provençal, qui convoite les terres de ses voisins; [C] Didace revient vers la maison qu'il observe tristement; [D] il y entre et dispute Phonsine qui a jeté aux poules le pain que, sous son regard sévère, elle avait laissé tomber par terre tout en se coupant le doigt. Seule la partie D est conservée dans les premiers épisodes du radioroman et du téléroman: la partie $B$ est reprise dans des épisodes subséquents et les parties $\mathrm{A}$ et $\mathrm{C}$, dans lesquelles Didace est seul16, sont éliminées. Dans les parties $C$ et $D$ du roman, le traitement des figures dans le roman est particulièrement riche, car celles-ci contribuent à la description d'Alphonsine et de la maisonnée des Beauchemin tout en permettant d'établir, nous le verrons, un léger décrochage par rapport à la «réalité » de la diégèse. Les figures, en effet, ne décrivent pas Phonsine

\footnotetext{
16 Genres destinés à un public de masse, le radioroman et le téléroman ne se prêtent pas facilement à la représentation de la solitude, notamment en raison de la difficulté que pose à la description l'intériorité d'un personnage.
} 
objectivement, mais subjectivement, c'est-à-dire telle qu'elle est vue par le père Didace.

Ayant déjà distingué les figures de la narration des figures du personnage, il faut encore ajouter que ces dernières, dans le roman, peuvent être attribuées au personnage dans un discours rapporté (je parlerai alors de figures rapportées) ou par focalisation (figures focalisées). Les figures rapportées mettent directement en scène les paroles ou la pensée du personnage. Dans les adaptations, nous l'avons vu, les figures sont principalement attribuées au personnage au moyen du discours direct. La palette du discours rapporté est cependant beaucoup plus étendue dans le roman et comprend notamment l'emploi du discours indirect libre. Vers la fin du second chapitre, Didace dispute Phonsine parce qu'elle jette du pain aux poules, puis, dans un long monologue intérieur en discours indirect libre, juge sévèrement sa bru et celle-ci fait, une fois de plus, l'objet d'une l'antithèse :

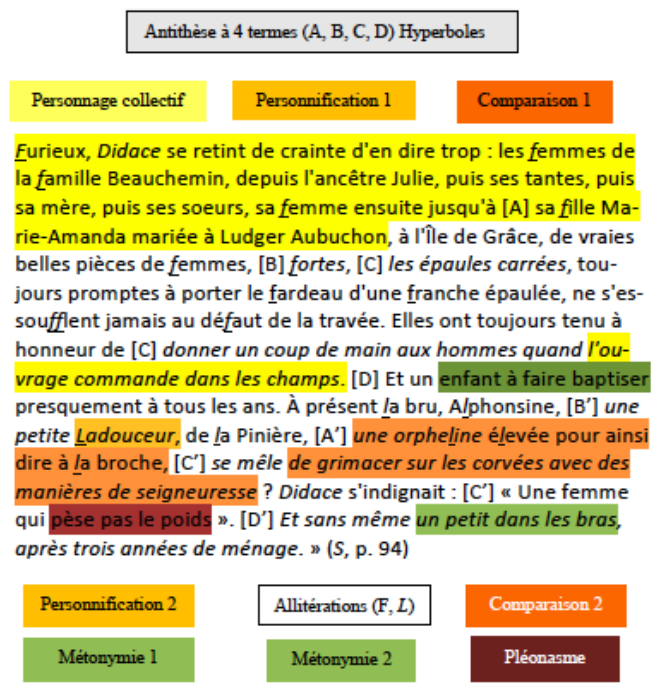


Diverses figures secondaires sont, on le voit, enchâssés à l'intérieur de l'antithèse. Or, la théorie des figures ne s'est guère penchée sur les interrelations des figures. Il s'agit pourtant d'un phénomène généralisé : les figures se combinent, s'agencent à l'intérieur d'une même phrase ou d'un texte suivi pour former des ensembles de figure, soit ce que j'appellerai des figures complexes. L'antithèse se renforce ainsi de nombreuses figures qui l'enrichissent, soit:

A) L'hyperbole: l'ensemble des qualités des femmes Beauchemin et des défauts d'Alphonsine possèdent un caractère hyperbolique qui accentue leur caractère antithétique.

B) Le personnage collectif: en amalgamant plusieurs générations de femmes en un type, cette curieuse figure s'oppose à Alphonsine qui est seule, orpheline et étrangère.

C) L'allitération («Retour d'une sonorité consonantique à intervalles rapprochés, plus spécialement à l'initiale des mots », Bacry, p. 278) : au plan sonore, l'allitération presque brutale des « $\mathrm{f}$ » dans la description des femmes Beauchemin tranche avec la douceur des «l $\mathrm{l}$ » associés à Phonsine.

D) Personnification (« Faire d'un être inanimé ou d'une abstraction un personnage réel », Dupriez, p. 344) : l'utilisation de l'adjectif «forte», dans le contexte de l'antithèse, réveille le caractère allégorique du patronyme de l'héroïne et transforme celle-ci en personnification de la Douceur.

E) Opposition de figures : des figures s'opposent parfois terme à terme, ainsi de la personnification («l'ouvrage commande ») et de la comparaison ("grimacer sur les corvées avec des manières de seigneuresse ») ou des deux métonymies 
de la grossesse ( « un enfant à faire baptiser presquement à tous les ans" / "sans même un petit dans les bras, après trois années de ménage »)

J'ajouterai enfin qu'Alphonsine fait elle-même l'objet d'une antithèse sociale (orpheline/seigneuresse) qui traduit son écartèlement (elle vient d'une classe défavorisée mais voudrait appartenir à une classe plus élevée) et son altérité par rapport au moyen terme socialement occupé par les femmes Beauchemin.

Dans le roman, la figure complexe que je viens de citer est assez clairement subjective. La trame des figures sert notamment à marquer - je développerai plus loin cette idée-que l'information est sujette à caution. Comment transposer cette figure? La scénariste aurait bien sûr pu transformer le discours indirect libre en discours direct par l'emploi d'un monologue intérieur, mais Guèvremont utilise avec parcimonie ce procédé. L'auteure suit plutôt, dans ses adaptations, deux stratégies différentes pour extérioriser ce monologue intérieur.

Se prêtant assez facilement à la transposition, le monologue intérieur de Didace est repris et développé sous une autre forme dans le radioroman. Guèvremont ajoute en effet, au début du second épisode, qui a lieu le lendemain matin, un échange verbal entre le Survenant et Didace qui reprend, en discours direct, une bonne partie de son monologue intérieur, antithèse comprise. Je citerai le passage au complet malgré sa longueur, sans toutefois marquer l'ensemble des figures :

SURVENANT Elle a pas l'air... ben... ben... forte, la p'tite mère! DIDACE Non, elle est pas forte su' l'ordinaire. Y s'en manque! 
SURVENANT C'est toujours pas le souper d'hier soir qui lui vaudrait un prix doré sur tranche. Stop. Ce que je voulais dire, c'est qu'elle a pas l'air forte de sa santé.

DIDACE La bru? J'te crés. Le moindre coup d'vent la jette à terre. Avec mépris et une légère indignation. Une créature qui pèse pas le poids! Pis même pas un p'tit dans les bras, après trois ans de ménage! Comment c'est que tu trouves ça, Survenant?

SURVENANT D'après ce que j'peux voir, vous l'avez pas en odeur de sainteté, père Didace?

DIDACE J'l'haïs pas, comme de raison. C'est la femme à mon garçon.

SURVENANT Seulement vous l'aimez pas gros. Rire. C'est comme rien, elle doit pas être de la paroisse?

DIDACE Elle est pas du Chenal. Mais a vient pas d'loin. C'est une p'tite Ladouceur de la Pinière, de l'autre bord de Saint-Joseph, en gagnant la grand' rivière. Stop. T'as pas connu son père!

SURVENANT J'serais bien en peine de le reconnaître si je l'rencontrais sur la rue.

DIDACE Un ivrogne d'la pire espèce! Le Survenant se rend au poêle. Théière de granit tirée sur le poêle. À c't'heure est orpheline.Avec mépris. Ç’a été élevée pour ainsi dire à la broche, d'un bord pis d'l'autre, à manger d'la sauce à la longue année. [...]

SURVENANT Pas du Survenant. C'est toujours pas de la faute de Phonsine si...

DIDACE C't'en quoi. À devrait faire un effort pour apprendre au lieu de grimacer su' toute comme si elle était la fille d'la seigneuresse de Berthier en personne!

SURVENANT Rire. Vot' garçon, lui, quoi c'est qu'il en dit?

DIDACE Amable? Y s'mire dans elle. Rapport qu'elle a enseigné la classe à des p'tits garçons pis à des p'tites filles, pis qu'elle a passé une couple de mois fille engagère en ville, $y$ pense qu'a connaît toute dans l'monde. Tout ce qu'a dit, tout ce qu'a fait, c'est beau et bon, comme parole d'évangile. Stop. Que j'aurais don voulu, au nom du ciel, que tu vinssis connaître les femmes de la famille Beauchemin!

SURVENANT Monologue intérieur. C'est étrange comme un homme, quand il vieillit, aime à parler de sa lignée! 
DIDACE Laisse-moi te dire que c'étaient des créatures dépareillées! Toutes. Toutes. De la première jusqu'à la dernière. Des vraies pièces de femmes. Carrées comme une maison de pierre. Ça prenait une bouchée. Mais ça travaillait. Aux champs, à faire les foins, comme les hommes. Sans vouloir les vanter.

SURVENANT Taquin. C'est pas à moi que vous ferez croire qu'il leur restait grand temps pour faire l'ordinaire et pour élever des grosses familles.

DIDACE Tu crés ça? Des bons repas chauds à tous les repas. Pis un p'tit à faire baptiser presquement à tous les ans. $\left(R R S, \mathrm{n}^{\circ} 2\right.$, 25 sept. 1962, p. 1-3)

Le développement de l'antithèse montre que, contrairement à un préjugé tenace, la langue de Guèvremont ne perd rien de sa saveur, de sa précision et de sa profondeur dans ses adaptations. Déjà riche, l'antithèse fait l'objet de nouveaux développements. Le dialogue se prête à merveille à ces ajouts. La première affirmation du Survenant sur Phonsine («Elle a pas l'air... ben... ben... forte, la p'tite mère! ») est ainsi mal comprise par Didace («Non, elle est pas forte su' l'ordinaire. ») Le Survenant, abondant d'abord dans le sens de Didace au moyen d'une hyperbole («C'est toujours pas le souper d'hier soir qui lui vaudrait un prix doré sur tranche »), le corrige («Ce que je voulais dire, c'est qu'elle a pas l'air forte de sa santé.»), ce qui donne lieu à de nouvelles figures («Le moindre coup d'vent la jette à terre. [...] Une créature qui pèse pas le poids! Pis même pas un p'tit dans les bras, après trois ans de ménage! »). Notons que tous ces nouveaux éléments seront par la suite intégrés dans l'antithèse globale d'Alphonsine et des femmes Beauchemin. Des figures secondaires de l'antithèse, quelque peu mystérieuses dans le roman, s'éclairent dans l'approfondissement qu'en propose le radioroman. La légèreté hyperbolique de Phonsine qui «pèse pas le poids» est complétée par une seconde hyperbole («le moindre coup 
d'vent la jette à terre ${ }^{17}$ ») et est, cette fois, clairement rattachée au fait que Phonsine n'a toujours pas d'enfants. Cette légèreté hyperbolique de Phonsine s'oppose à la comparaison des femmes-maisons du Chenal du Moine ("Carrées comme une maison de pierre ») qui sont, elles, lourdes et enracinées. La vision antithétique qu'ont de Phonsine Didace et Amable participe également de l'approfondissement de l'antithèse. Je reviendrai plus loin sur le rôle du Survenant qui rend manifeste la partialité du père Didace et de ses figures.

Dans le téléroman, Guèvremont choisit une autre voie pour transposer l'antithèse. La perte de la narration la forçant de nouveau à déléguer quelques-unes de ses fonctions à des personnages, c'est Beau-Blanc qui, d'extrêmement effacé qu'il était dans l'incipit du roman, prend maintenant en charge une fonction importante de la narration : c'est lui qui présente les divers personnages (au Survenant comme aux auditeurs). Ainsi, dans le téléroman, c'est lui qui reprend l'information contenue dans le monologue intérieur du père Didace. Montrant un portrait de Mathilde, Beau-Blanc fait son apologie :

(AVEC ADMIRATION) Elle! mmm! une vraie femme de maison! Jamais un grain de poussière nulle part. On pouvait manger à terre, tant que c'était net partout. (Pause) Puis... à tous les ans... là (IL PREND LE PREMIER OBJET À SA PORTÉE SUR LA TABLE, SOIT LA SALIÈRE OU LE SUCRIER, ET FAIT LE GESTE D'UNE FEMME QUI BERCE SON ENFANT PENDANT QUELQUES INSTANTS) Ah! ah! ah! (PAUSE) Ah! ah! ah! (REPLACE L'OBJET SUR LA TABLE) Elle passait pas une année sans acheter $^{18}$. (TRS, no 1,2 nov. 1954, p. 18)

\footnotetext{
17 L'image s'oppose de façon très précise aux femmes Beauchemin du roman qui «ne s'essoufflent jamais au défaut de la travée ». Cette première image disparaît toutefois du radioroman.

18 L'affirmation est curieuse puisque Mathilde n'a eu que trois enfants.
} 
Guèvremont, dans son téléroman, prend donc à la lettre la maxime américaine "Don't tell it, show it » (le genre s'y prête, bien entendu)... Le «type » des femmes Beauchemin s'incarne dans le personnage de Mathilde, beaucoup plus présent maintenant que dans l'incipit du roman. Beau-Blanc va en effet décrocher du mur le portrait de la défunte. Notons que l'effort pour montrer s'accompagne du désir de symboliser: Guèvremont introduit en effet, par une indication scénique, une métaphore visuelle. Explicite dans le roman, l'antithèse devient implicite dans le téléroman (Guèvremont note dans la didascalie qui suit la réplique de Beau-Blanc que Phonsine est «mal à l'aise ») et perd un peu de son caractère accusateur pour la bru. En revanche, l'information gagne en objectivité, car le monologue intérieur s'extériorise et change de voix (BeauBlanc est un tiers parti). Toutes ces minuscules variations s'accumulent et transforment de façon importante la présentation du personnage de Phonsine dans les adaptations. En même temps, il est évident que l'amenuisement de la narration extradiégétique qui a lieu dans les adaptations prive la romancière d'un «outil» important qui lui permet d'exprimer l'intériorité et la subjectivité de ses personnages, soit ce que Genette a appelé la focalisation et que Kaempfer et Zanghi définissent comme « le mode d'accès au monde raconté, selon que cet accès est, ou n'est pas, limité par un point de vue particulier $^{19}$ ».

Les figures du personnage peuvent, comme on l'a vu, prendre place dans un discours rapporté, mais elles peuvent également être présentes dans un segment de texte focalisé sur un personnage. La figure devient alors narrativement hybride,

${ }^{19}$ Kaempfer, Jean \& Zanghi, Filippo (2003). 
car elle appartient simultanément à la narration et au personnage. Les figures focalisées sont employées dans ce que Genette appelle une focalisation interne et sont assez clairement rattachées au champ de conscience d'un personnage. Rappelons que, dans la focalisation interne, le narrateur ne raconte que ce que sait, voit, ressent un personnage donné (focalisation internefixe), ou plusieurs personnages successivement (focalisation interne variable); il peut également, à travers le même procédé, revenir sur un même événement selon les points de vue de personnages différents (focalisation interne multiple) (Genette, 1972, p. 206207).

Dans le cas des figures focalisées, toutefois, le champ de conscience n'est pas représenté (comme dans les figures rapportées) mais fait plutôt l'objet d'une traduction, d'une figuration. Clairement extérieures au champ de conscience du personnage, les figures sont données par la narration comme un équivalent de celui-ci, qu'elles «traduisent» ou «symbolisent ». Dans le second chapitre, par exemple, diverses figures hyperboliques servent ainsi à rendre compte de l'humeur sombre du père Didace :

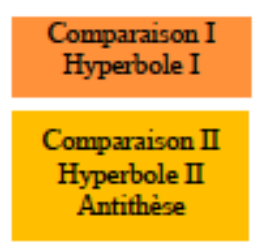

Depuis la mort de Mathilde, sa femme, non seulement Didace recherchait les occasions de s'éloigner de la maison, mais il la fuyait, comme si le sol lui eût brûlé les pieds, comme si les choses familières, jadis hors de prix, à ses yeux, s'y fussent ternies et n'eussent plus porté leur valeur. ( $S$, p. 93) 
La «figuration» du champ de conscience permet à la romancière de traduire en mots ce qui échappe normalement au langage dans la psyché humaine (impressions, sentiments, désirs). Il s'agit d'un emploi fort subtil mais assez courant des figures dans le roman.

À cheval entre la voix de la narration et la voix du personnage, les figures focalisées sont, par essence, hybrides, dialogiques (au sens où l'entend Laurent Jenny en s'inspirant des travaux de Ducrot $^{20}$ : «il y a dialogisme dès que deux voix se disputent un seul acte de locution $\left.{ }^{21} »\right)$. Cette hybridité des figures focalisées les rend fort difficiles à adapter à d'autres médias et elles tendent à disparaître ou à être traduites en langage non figuré ${ }^{22}$. Didace exprime ainsi directement sa vision dysphorique de la ferme : "Le fournil est à la veille de tomber en ruine. Y a l'étable à recouvrir en neuf » $\left(R R S, \mathrm{n}^{\circ} 1,24\right.$ sept. 1962, p. 5). De même, l'humeur sombre du père Didace n'est pas décrite de l'intérieur, mais de l'extérieur, par Amable et Phonsine :

PHONSINE Le souper est dressé. Tu fais mieux de descendre. Ton père est pas de bonne humeur.

AMABLE C'est pas nouveau. Depuis que la mère est morte, il trouve à r'dire sur tout.

PHONSINE Oui, mais aujourd'hui, je sais pas, il est pas comme de coutume. Il a l'air... tanné, on dirait. Tanné pour vrai. (RRS, no 1, 24 sept. 1962, p. 4)

\footnotetext{
20 Oswald Ducrot, «Esquisse d'une théorie polyphonique de l'énonciation », Le dire et le dit, Paris, Minuit, 1984. p. 171-233.

21 Laurent Jenny (2003).

22 L'antithèse temporelle «avant/ maintenant» est toutefois reprise par le père Didace : « Ça [les exploits de chasse], c'était avant que la mère Mathilde vinssît mourir. Dans le bon temps quand j'avais de l'aide... » Cette antithèse passéiste traverse par ailleurs tout le roman (RR1).
} 
Si la perte de la focalisation nuit à l'expression de l'intériorité du père Didace, qui perd en nuance et en profondeur, elle oblige toutefois l'auteure à varier les points de vue. La subjectivité d'Amable et de Phonsine, globalement absente du premier roman, trouve ainsi à s'exprimer. Cette dernière, dans le téléroman, se défendra même "avec rancœur » des attaques de Didace: "Il m'a encore appelée... la petite Ladouceur, comme si j'étais pas ta femme! Comme si c'était d'ma faute qu'on n'ait pas d'enfant! » (TRS, no 1, 2 nov. 1954, p. 12)

L'une des caractéristiques génériques des adaptations est de clarifier ce qui est objectif et ce qui est subjectif, ce qui n'est pas toujours le cas dans le roman. Le rapport des figures et du champ de conscience du personnage, explicite dans les figures rapportées, est en effet souvent implicite dans les figures focalisées du roman :

Sans hâte il racla ses bottes au seuil, tout en jetant un coup d'œil à l'intérieur de la maison. Il pouvait voir une bonne partie de la pièce principale, à la fois

Comparaison Hyperbole

Antithèse cuisine et salle. Les rideaux sans apprêt pendaient comme des loques aux fenêtres et dans les deux chambres du bas, la sienne et celle du jeune couple, les lits de plume, autrefois d'une belle apparence bombée, maintenant mollement secoués, s'affaissaient au milieu ${ }^{24}$. (S, p. 93)

Ainsi, si l'une des fonctions de la narration romanesque consiste à "gérer » ce qui est objectif et ce qui est subjectif, la focalisation, dans le roman, cause (ou permet) un certain flou dans l'énonciation. La vision subjective qu'a Didace de Phonsine et de la maisonnée risque ainsi fort d'être perçue par le lecteur comme une réalité objective attribuées à la narration. Il est par 
exemple impossible de savoir si la description suivante de Phonsine est objective ou subjective :

Comparaison Hyperbole
En entrant, Didace trouva la table à moitié mise et Phonsine affaissée sur une chaise. Frêle, les épaules et les hanches étroites, avec ses cheveux tressés en deux nattes sur le dos et ainsi abandonnée à ellemême, elle avait l'air d'une petite fille en pénitence. (S, p. 93)

Il est tout aussi difficile de déterminer si la comparaison est une figure de la narration ou une figure du personnage. L'enjeu est pourtant de taille, car l'évaluation de ce qui est objectif et ce qui est subjectif dans ces chapitres liminaires s'avère déterminante pour la perception que le lecteur aura des personnages de Phonsine et de Didace.

Alain Rabatel a étudié divers procédés de construction textuelle du point de vue. L'un des procédés les plus significatifs qu'il dégage est l'« opposition entre les premiers et deuxièmes plans du texte » (p. 25). Dans un extrait déjà cité, la première phrase (au passé simple) forme ainsi le premier plan duquel se dégage, dans les deux phrases suivantes (à l'imparfait), un second plan :

Selon Rabatel, cette hiérarchisation des plans, en provoquant un «décrochage énonciatif» (p. 25), «fournit un outil commode permettant de distinguer ce qui est objectif de ce qui est subjectif » (p. 31). Les figures, dans l'extrait, apparaissent au second plan et contribuent à souligner la subjectivité du point de vue (on pourrait les considérer comme des "subjectivèmes", c'est-à-dire "des marques d'appui dans l'embrayage du point de vue du personnage » [Rabatel, p. 83]). 
Il faut cependant, je crois, aller encore plus loin, car l'étude des figures révèle une hiérarchisation similaire des plans au niveau de la fiction. Elles constituent en effet des fictions dans la fiction, des microfictions : « [L]a métaphore, et plus généralement la figure, ou du moins les figures de substitution comme la métaphore ou la métonymie, l'antiphrase, la litote ou l'hyperbole, sont des fictions verbales et des fictions en miniature. » (Genette, 2004, p. 19) Les figures instaurent en effet des microfictions dans lesquelles les personnages sont littéralement mis en scène :

\section{Fiction prin- cipale}

Microfictions
Depuis la mort de Mathilde, sa femme, non seulement Didace recherchait les occasions de s'éloigner de la maison, mais il la fuyait, comme si le sol lui eût brûlé les pieds, comme si les choses familières, jadis hors de prix, à ses yeux, s'y fussent ternies et n'eussent plus porté leur valeur. (Je souligne) (S, p. 93)

Le comparant "comme» instaure non seulement une figure mais une fiction. On peut donc discerner, au premier plan, la fiction principale et, au second plan, deux microfictions. Les figures provoquent ainsi un « décrochage » fictionnel, voire des fluctuations dans la fiction : les métafictions sont en effet plus fictives que la fiction principale. Comme le décrochage énonciatif dont parle Rabatel, le décrochage fictionnel instauré par les figures permet de distinguer ce qui est objectif de ce qui est subjectif.

$\mathrm{Au}$ contraire du roman, qui est, on l'a vu, un genre à subjectivité variable, le radioroman et le téléroman sont des genres dans lesquels la subjectivité est fixe, car ceux-ci 
répartissent sur des «canaux» différents l'objectif et le subjectif: les figures du personnage et les fictions subjectives qu'elles créent sont clairement attribuées au personnage en discours direct tandis que la réalité objective est constituée par l'ensemble de ce que l'auditeur entend (dans le cas du radioroman) ou voit et entend (dans le cas du téléroman). Cette « clarification » de l'énonciation présente des défis particuliers.

Il en résulte que les trois genres présentent, dans leurs chapitres ou leurs épisodes liminaires, différentes visions d'Alphonsine. Le roman, par la focalisation sur Didace faite dans le second chapitre, dresse le portrait le plus sévère de Phonsine et de la maisonnée des Beauchemin. Même si les figures témoignent de la subjectivité de ce portrait, celui-ci se mêle suffisamment à la narration objective pour former le point de vue dominant du roman (ce qui ne sera plus le cas dans la suite de ce roman, Marie-Didace, qui épousera globalement le point de vue de Phonsine).

Dans les épisodes liminaires du radioroman, ce point de vue dominant disparaît avec la focalisation. Des trois genres, le radioroman est le plus subjectif parce que presque toute l'information passe par les personnages. Cette subjectivité est en même temps garante de l'objectivité du genre: l'auditeur entend tout ce qui se passe chez les Beauchemin comme s'il y était lui-même. Il demeure, il est vrai, une narration objective et celle-ci peut, dans certains cas, donner des informations importantes et contribuer à forger l'identité des personnages comme c'est le cas dans le premier épisode : «La maison des Beauchemin... vers six heures... comme Phonsine, la bru du père Didace, se berçait rêveusement... » (RRS, no 1,24 sept. 1962, p. 6) La légèreté et la paresse de Phonsine, alors que le 
souper n'est pas fait, conforteront plus tard la position de Didace sur sa bru. Plus neutre, le roman présente Phonsine, à l'entrée de Didace dans le second chapitre, comme étant « affaissée » sur une chaise.

Le fait que Phonsine apparaisse la première dans l'incipit des adaptations constitue un changement de taille, car ce n'est plus Didace qui «contamine» la vision objective de Phonsine, mais le contraire. La venue de Didace, dans le radioroman, est menaçante : " [Phonsine à Beau-Blanc] Affolée. Ah! mon doux, v'là mon beau-père. Le poêle qui tire pas. Cours, Beau-Blanc, cours vite me chercher une brassée de p'tit bois fin, pendant que je mets la table » $\left(R R S, \mathrm{n}^{\circ} 1,24\right.$ sept. 1962, p. 2). Phonsine a ainsi l'avantage d'occuper le terrain avant Didace dont l'apparition est précédée d'une série de commentaires qui soulignent sa sévérité et sa mauvaise humeur. On a souvent comparé la focalisation au jeu d'une caméra. Le " point de vue », on le voit, ne disparaît pas tout à fait car Guèvremont aurait pu choisir de placer son micro avec Didace, à l'extérieur. Notons également que le radioroman est plus subjectif que le téléroman parce qu'il n'occupe pas le canal de la vue. Il laisse ainsi, comme on l'a souvent dit, une plus grande place à l'imagination. Comme Phonsine, la cuisine des Beauchemin fait l'objet de spéculation de la part des divers personnages, et c'est à l'auditeur, comme au lecteur, de décider si la cuisine des Beauchemin est telle que la voit Didace dans sa mauvaise humeur.

Dans le roman, les allers retours entre la subjectivité des personnages et l'objectivité de la narration permettent d'éclairer, de diverses façons (appui, explications, ironie, mise à distance, etc.), le discours des personnages. Il pourrait sembler, 
à première vue, que l'amenuisement de la narration dans le radioroman cause un aplatissement généralisé de la subjectivité, une sorte de démocratisation faisant en sorte que toutes les subjectivités se valent. À y regarder de plus près, toutefois, il apparaît que Guèvremont peut déléguer aux personnages la fonction de faire valoir un point de vue ou de le questionner. La longue mise en antithèse de Phonsine par Didace que nous avons citée plus haut sera ainsi éclairée et mise à distance par le Survenant. Toutes ses répliques viennent en effet subtilement déconstruire l'antithèse de Didace. Il met en effet en relief les outrances et les non-dits de sa vision, et le force à avouer ses préjugés :

SURVENANT D'après ce que j'peux voir, vous l'avez pas en odeur de sainteté, père Didace?

DIDACE J'l'haïs pas, comme de raison. C'est la femme à mon garçon.

SURVENANT Seulement vous l'aimez pas gros. Rire. C'est comme rien, elle doit pas être de la paroisse?

Objectif, le Survenant se garde de répondre lorsque le père Didace réclame son approbation : "Une créature qui pèse pas le poids! Pis même pas un p'tit dans les bras, après trois ans de ménage! Comment c'est que tu trouves ça, Survenant? » Il défend même Phonsine lorsque Didace lui fait reproche d'être née dans un milieu défavorisé : "C'est toujours pas de la faute de Phonsine si...». Guèvremont met même à profit le monologue intérieur pour montrer la partialité de Didace (« Monologue intérieur. C'est étrange comme un homme, quand il vieillit, aime à parler de sa lignée! ») Enfin, le Survenant force Didace à élaborer une autre antithèse, qui montre Phonsine sous un regard entièrement différent : SURVENANT Rire. Vot' garçon, lui, quoi c'est qu'il en dit? DIDACE Amable? Y s'mire dans elle. Rapport qu'elle a enseigné la classe à des p'tits garçons pis à des p'tites filles, pis qu'elle a passé une couple de mois fille engagère en ville, y pense qu'a connaît toute dans l'monde. Tout ce qu'a dit, tout ce qu'a fait, c'est beau et bon, comme parole d'évangile. 
Le rire du Survenant joue un rôle crucial dans cette mise à distance.

Dans le téléroman, l'objectivité et la subjectivité passent par un canal différent, car la gestion des premiers et des seconds plans repose sur une opposition entre ce qui est objectivement montré et ce qui est subjectivement dit $^{23}$. La cuisine Beauchemin, qui illustre les qualités de ménagère de Phonsine, ne peut plus être laissée à l'imagination du lecteur. L'auteure, le réalisateur et le décorateur doivent statuer sur celle-ci. Guèvremont, dans l'incipit, montrera ainsi Phonsine en train de nettoyer son plancher. C'est d'ailleurs dans l'incipit du téléroman que celle-ci apparaît sous son meilleur jour.

Filmé en direct à chaque semaine, le téléroman, à l'époque de Guèvremont, était, peut-être encore plus qu'aujourd'hui, une grosse machine. Le radioroman, relativement artisanal, se plie assez facilement aux exigences de l'auteure. Dans le téléroman, au contraire, Guèvremont doit non seulement adapter son roman au nouveau média, mais elle doit s'adapter aux décisions prises par le réalisateur et par l'équipe de production. Ainsi, la maigreur de Phonsine et toutes les figures qui y sont associées disparaîtront des scénarios pour s'adapter aux rondeurs de son interprète, Suzanne Langlois.

\section{Transposition et interprétation des figures}

Consacré à la voisine des Beauchemin, Angélina Desmarais, le troisième chapitre du roman raconte la «saisie» de ce

\footnotetext{
23 Exception faite du langage corporel. Notons également que les fictions télévisuelles contemporaines (Six Feet Under, par exemple), en intégrant des microfictions à la fiction principale, problématisent cette opposition.
} 
personnage : « [...] à la vue du Survenant qu'elle ne connaissait pas, elle s'arrêta, saisie » $(S$, p. 99). Les différents sens du verbe " saisir » expriment tous avec force la " possession » amoureuse $\mathrm{du}$ personnage, sa «capture» presque mythologique par le Grand-dieu-des-routes. En outre, l'image du saisissement revient plus loin dans le roman pour décrire la passion d'Angélina pour la musique des mots: "Aux passages mystiques "couronne de vie", "enfants de la lumière", "le juste fleurira comme le palmier", "doux hôte de l'âme", elle s'arrêtait, saisie, plus attentive à la musique qu'au sens des mots. Sûrement Dieu l'appelait à Son service. Comment expliquer autrement l'éblouissement intérieur qui la gagnait? » $(S$, p. 121122) Guèvremont n'a jamais mieux exprimé que dans ce passage son amour pour les figures, et notamment pour la métaphore, qui jouent un rôle primordial dans son écriture. Or, la transposition des métaphores dans les adaptations s'avère, on l'a dit, particulièrement difficile. Dans le troisième chapitre, la personnification du vent et la métaphore de la cloche de la Pèlerine expriment symboliquement la « saisie » d'Angélina, et l'on verra les efforts considérables que devra déployer l'auteure pour les transposer dans d'autres médias.

Le vent, comme je l'ai montré ailleurs (Décarie 2001), occupe en effet une place centrale dans le troisième chapitre :

Le vent, un vent d'octobre, félin et sournois, qui tantôt faisait le mort, comme muet, l'œil clos, griffes rentrées, allongé mollement au ras des joncs secs, et insoucieux de rider même d'un pli la surface de l'eau, maintenant grimpé au faîte des branches, secouait les arbres à les déraciner. En deux bonds il fonça sur la route, souleva la poussière à pleine rafale, entraîna les feuilles sèches dans une danse folle et poussa même, hors de son chemin, un passant. Puis il harcela la rivière qui écumait, moutonneuse, et colla les embarcations à la grève, ébranla les 
toits des vieux bâtiments, ouvrit les portes à deux battants et courut aux champs coucher un dernier regain : un vent du diable, hurlant à la mort. Il fit rouler un bidon jusqu'au bas du talus. (S, p. 99)

L'épisode débute ainsi par une longue personnification du vent qui préfigure non seulement le tohu-bohu que provoquera l'arrivée du Survenant au Chenal du Moine, mais aussi la passion qui emportera Angélina. Celle-ci, en effet, est littéralement attaquée par le vent juste avant sa rencontre avec le Survenant :

Personnification

Avant de s'engager dans le sentier oblique conduisant à la maison des Beauchemin, Angélina Desmarais s'arrêta près de la haie vive et chercha son souffle. À marcher seule, elle trouvait la route longue et, au tournant de la montée, le vent embusqué dans les saules l'avait assaillie à la gorge et quasiment jetée par terre. $(S$, p. 97$)$

Guèvremont, par la personnification $d u$ vent, fait coexister la morale et son envers : la force sauvage, sexuelle et inconsciente symbolisée par le coup de vent annonce, prépare et symbolise la saisie d'Angélina. L'écriture de Guèvremont est hautement symbolique, et même les phrases employées au sens propre peuvent se révéler avoir un double sens : ainsi de la route qui semble longue à l'héroïne dans sa solitude, ainsi du « souffle» («force qui anime, inspire, crée ») qu'elle cherche et qui lui apparaîtra sous forme de vent. Or, à plusieurs reprises dans le roman, la paronomase rapproche le Survenant et le vent 


\section{- [...] J'aurais jamais dû faire parler ce grand fou} de Venant.

Puis elle écouta : pour toute réponse, un ronflement d'homme ivre, le sifflement du vent. (S, p.204) Angélina tourna légèrement la tēte. [...] la figure coParonomases lorée du. Survenant, les cheveux roux au vent, tranchait sur fe rideau de ciel pur.» (S, p. 218)

La comparaison se prête également à ces rapprochements : "Après s'être dandiné mollement de bord en bord de l'embrasure, le Survenant se décida à franchir le seuil de la porte, levant les pieds de façon exagérée. Comme un arbre à tous les vents, il chancelait » $(S$, p. 198). Le récit, par diverses comparaisons, métaphores et développements thématiques, travaille à combler l'espace séparant le vent du Survenant. «Fend-le-vent», comme l'appelle Phonsine ( $S$, p. 112), « rentre en bourrasque dans la maison» $(M-D$, p. 209), ouverte par le père Didace à tout venant, à tout vent.

À moins de verser dans le fantastique ou dans le symbolisme à la Maeterlinck, la personnification est impossible à incarner à la radio ou à l'écran. Une première lecture de l'adaptation radiophonique $\mathrm{du}$ troisième chapitre peut d'ailleurs laisser croire que Guèvremont déclare forfait: au début du neuvième épisode, elle renonce en effet à mettre directement en scène la personnification du vent et se contente de donner au narrateur les lignes mêmes de son roman citées plus haut. Elle ajoute toutefois une réplique qui fait directement le lien entre Venant et le Vent :

NARRATEUR Mais est-ce bien seulement le vent qui empêche Angélina Desmarais d'avancer? Stop. Depuis dimanche aprèsmidi elle ne fait que songer à l'étranger qui l'a secourue sur la 
route, après qu'Odilon Provençal l'eut abandonnée. (RRS, nº 9, 4 oct. 1962, p. 1)

Cette réplique fait surtout le lien avec une scène inédite, inventée pour le radioroman, qui constitue la véritable transposition du coup de vent. Guèvremont, pour exprimer la saisie, utilise une scène qui n'est pas sans rappeler celle qui arrive vers la fin du roman, où le cheval d'Angélina s'emballe à l'approche d'une automobile alors que le Survenant se fait dire la bonne aventure par une jeune gitane. Dans le radioroman, la nouvelle scène, dans le huitième épisode, exprime, simultanément la passion amoureuse naissante d'Angélina pour le Survenant et la jalousie d'Odilon Provençal, le rival du Survenant. En effet, c'est Odilon qui, suite au refus d'Angélina d'envisager leur mariage, cause l'emballement du cheval :

ODILON Ça revient au même. De plus en plus en colère. Qui c'est que ça t'prend d'abord? Un gâs d'la ville? Ou ben don... un chéti garnement... comme le SURVENANT!

ANGÉLINA Perdue. Quel Survenant?

ODILON Il saute en bas de la voiture. Quiens! Le v'là justement sur la route! Arrange-toi avec!... Corneille, va! Fo

ANGÉLINA Le cheval part au galop. Odilon! Me laisser de même sans m'avertir. Arrié! Le Blond. Arrié!

SURVENANT Ouô don! Il a sauté à la bride du cheval. Le cheval ralentit petit à petit.Ayez pas peur! Ayez pas peur!

ANGÉLINA C'est pas tant la peur! Mais j'suis restée saisie. Me faire un affront semblable en plein cœur du village.

SURVENANT Pour dire le vrai, votre cavalier vous a faussé compagnie un peu vite, la belle! Mais les chicanes d'amoureux... durent peu.

ANGÉLINA Promptement. C'est pas mon cavalier!

SURVENANT Vous protestez trop vite!

ANGÉLINA Non, non, quand je vous le dis!

SURVENANT Je peux aller vous reconduire chez vous? 
ANGÉLINA C'est pas la peine. D'ailleurs j'ai l'habitude de mener un cheval. Seulement, comme je vous le disais tantôt, je suis restée saisie. Passez-moi les guides. (RRS, no 8,3 oct. 1962, p. 7)

Le double emploi du mot « saisie » (que le narrateur du radioroman reprendra dans l'épisode suivant pour décrire le coup de foudre d'Angélina) montre clairement la parenté des épisodes et la redondance symbolique de leur fonction. Le terme «cavalier » utilisé par le Survenant fait également le lien entre les lexiques équestre et amoureux.

Dans le roman, la fascination d'Angélina pour le Survenant s'exprime par une série de métaphores : la main du Grand-dieu-des-routes devient une étoile, sa chevelure, un feu de forêt: "En l'apercevant tantôt, elle avait songé : "C'est pire qu'un feu de forêt" » $(S$, p. 105). Ces courtes métaphores se transposent très facilement dans le discours du personnage : «C'est de lui voir la chevelure. Si on dirait pas... un vrai feu d'forêt » (TRS no 2, 9 novembre 1954, p. 7). Il en va tout autrement des métaphores plus longues :

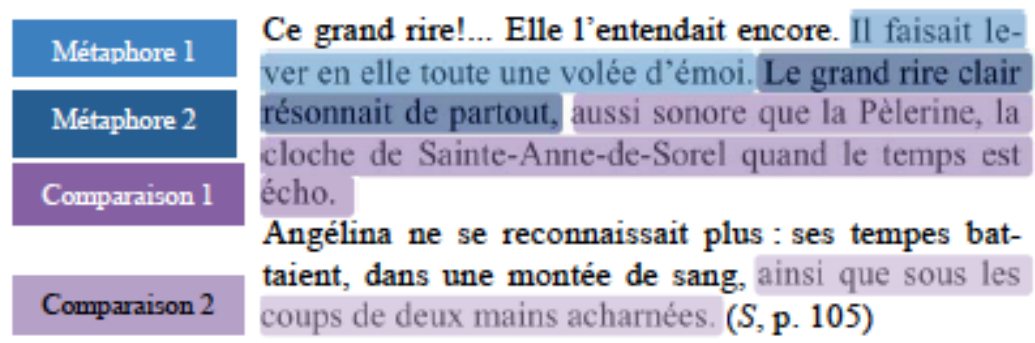


L'ensemble de ces figures forme une "figure complexe ${ }^{24}$ dont l'unité est à chercher dans le lexique de la cloche qui essaime à travers tout le passage («volée», « résonnait de partout», « cloche », «battaient», « coups »). L'un des aspects les plus fascinants de cette figure complexe est le fait qu'elle unit les deux personnages : la métaphore 1 et la comparaison 2 décrivent en effet le violent émoi d'Angélina tandis que la métaphore 2 et la comparaison 1 s'appliquent au rire du Survenant. Cette fusion amoureuse des personnages revient d'ailleurs plus loin dans le roman avec l'image de l'harmonie rythmique du cœur d'Angélina, du rire du Survenant et de l'angélus de la Pèlerine :

Son cœur battait fort contre sa poitrine comme pour s'en échapper et courir au devant du bonheur. Elle le comprima à deux mains et écouta : dans le midi bleu, un grand rire clair se mêlait à la cloche de l'angélus et les deux sonnaient l'allégresse à pleine volée » $(S$, p. 218).

Dans le téléroman, seule la comparaison est reprise, en discours direct, par Angélina :

ON ENTEND LE RIRE DU SURVENANT.

ANGÉLINA Ce grand rire! Comme la Pèlerine, la cloche de Sainte-Anne, de Sorel, quand le temps est écho! (TRS, no 2,9 novembre 1954, p. 7)

La métaphore, on le voit, est réduite à sa plus simple expression et n'est plus à même d'exprimer la fusion d'Angélina et du Survenant. Guèvremont, toutefois, ajoute une série de nouvelles scènes dans le but de transposer celle-ci. Après avoir associé le rire du Survenant à la Pèlerine, l'auteure associe Angélina au son de l'harmonium qui se trouve chez elle:

24 Pour une analyse détaillée de cette figure, voir Décarie 2006. 
Le couvercle de l'harmonium est rabattu... Elle [Angélina] le soulève un peu. Le rabat. Le soulève et effleure les notes rêveusement. Puis entendant quelqu'un le rabat. (TRS, $\mathrm{n}^{\circ} 2$, 9 nov. 1954, p. 13)

Ce quelqu'un est Odilon Provençal, qui lui demande de lui accorder la faveur de la veillée en vue d'un mariage. Elle refuse, mais un mariage d'une autre sorte a toutefois lieu, ou plutôt un accord, une harmonie :

Odilon sort. Angélina s'en va à l'harmonium, soulève le couvercle et caresse les notes. Elle en touche quelques-unes qui se mêlent à la cloche de Sainte-Anne de Sorel... (TRS, no 2 , 9 nov. 1954, p. 15)

On peut également lire cette image comme un petit clin d'œil au travail d'adaptation. Elle décrit en effet très bien la polyphonie et les jeux d'échos que les multiples variations intermédiatiques introduisent dans le cycle du Survenant.

Les figures du troisième chapitre montrent à quel point le travail d'adaptation est en même temps un exercice de relecture et de réécriture qui se rapproche souvent du commentaire, voire, dans le cas des figures, d'une autointerprétation. Des procédés d'écriture qui n'étaient sans doute pas pleinement conscients au moment de l'écriture du roman apparaissent ainsi en pleine lumière. Il existe par exemple une curieuse figure de style, assez proche de l'isolexisme par dérivation dont parle Bernard Dupriez («variations lexicales : sous différents vocables, on a toujours la même lexie », Dupriez, p. 266), qui traverse l'œuvre romanesque de Guévremont, et qui établit une relation entre les noms du personnage principal - survenant, Survenant, le Survenant, Venant - et les variations lexicales du verbe "venir ». Voici un florilège de la figure en question, que j'ai nommée « venance » (voir Décarie 2001) : 
Venant vint sur le point d'ajouter [...] (S, p. 110);

[...] la première voiture à revení au chenal après la grande messe ramena le Survenant ( $S$, p. 167);

À l'avenir, tâche donc de te comporter comme un homme, Survenant. (S p. 210):

[...] il devient davaptage le Venant à Beauchemin [...] (S, p. 264);

Venant prévint l'Étrangleur [...] (S, p. 253);

Quand elle parvint au seuil de la porte, Venant lui demanda [...] (S. p. 266);

Les prévenances du Survenant lui manquaient. ( $M-D$, p. 93);

Tu nieras toujours pas que 1e Survenant était prévenant comme on en voit rarement?

$(M-D, .126)$

Ces rapprochements, comme je l'ai montré dans mon article, jouent un rôle non négligeable dans le développement $\mathrm{du}$ personnage et $\mathrm{du}$ roman. L'association «Survenant/ revenant » s'inscrit par exemple en sous-main dans la relation des divers personnages avec l'étranger :

Le Survenant, sans passé, sans nom, sans attaches, permet à chacun de projeter sur lui ses désirs ou ses craintes; permet aux pulsions et aux souvenirs d'affleurer, de venir, de revenir. Son étrangeté absolue mène paradoxalement au fantasme d'une parenté, d'une familiarité: à chaque personnage son Survenant, son «revenant». (Décarie, 2001, p. 370)

Ces rapports, toujours implicites dans les romans, deviennent explicites dans le radioroman : 


\begin{tabular}{|c|c|}
\hline PHONSINE & Avec mépris. Un survenant... Ricane. \\
\hline SURVENANT & $\begin{array}{l}\text { Oui un survenant pour les gens du Chenal du Moi- } \\
\text { ne. Mais ... ma1s (s'amuse à apeurer Phonsine) } \\
\text {...qui t'dit que je ne suis pas plutôt... un... reve- } \\
\text { nant, Oui un revenant... }\end{array}$ \\
\hline PHONSINE & Fais-moi pas peur, Survenant. On entend le vent. \\
\hline SURVENANT & As-tu entendu? \\
\hline PHONSINE & Le vent? \\
\hline SURVENANT & Je sais pas. Stop. Écoute! Le vent augmente. \\
\hline PHONSINE & Voyons donc! C'est les liards qui s'lamentent. \\
\hline SURVENANT & $\begin{array}{l}\text { Es-tu sûr que je suis pas revenu de l'autre monde } \\
\text { pour expier quelque crime que } j \text { 'aurais commis de } \\
\text { mon vivant? }\end{array}$ \\
\hline PHONSINE & Arrête donc de m'faire des peurs semblables... \\
\hline SURVENAN & $\begin{array}{l}\text { Quoi, t'as jamais entendu parler des âmes errantes } \\
\left.\text { sur la terre... (RRS, } \mathrm{n}^{\circ} 22,23 \text { oct. } 1962 \text {, p. } 1-2\right)\end{array}$ \\
\hline
\end{tabular}

L'association Survenant-revenant s'enrichit ici de la relation purement sonore de Venant et du vent. Guèvremont, dans le radioroman puis dans le téléroman, recommence, sous une autre forme, ce qu'elle a déjà achevé. Dans une entrevue avec Louis Pelletier-Dlamini, elle s'est expliquée sur ce travail de parachèvement, et sur sa valeur :

- Mais, Madame, ne croyez-vous, pas que le succès d'un roman entraînant les succès radiophoniques et télévisés aient nui à votre évolution littéraire?

- À mon évolution littéraire proprement dite, non! Je n'ai jamais cessé d'écrire et de lire depuis. Et je crois que chaque paragraphe bien équilibré, quelle que soit sa destination, parachève une forme de style, fournit une autre lueur sur ce terrible métier qu'est la Littérature. (1967, p. 84)

Anne Besson montre que la forme du cycle repose sur le fait de permettre la « coexistence entre unité et discontinuité, répétition et diversité» (p. 13). Pour étudier le Cycle du Survenant, le critique doit lui-même respecter cet équilibre et éviter deux types de réduction de l'œuvre de Guèvremont. La 
première réduction, la plus courante, consiste à ne pas suffisamment tenir compte de l'homogénéité de l'œuvre en séparant trop radicalement l'œuvre littéraire et l'œuvre médiatique. Les raisons et les présupposés de l'exclusion des genres médiatiques du domaine de la littérature sont trop complexes et trop nombreux pour être abordés ici. Toutefois, le travail d'orfèvre opéré par Guèvremont pour faire passer ses figures du roman au radioroman et au téléroman témoigne avec éloquence de la littérarité des écritures radiophonique et télévisuelle.

La seconde réduction consisterait à ne pas suffisamment tenir compte de l'hétérogénéité de l'œuvre. Une des limites de la théorie des figures de style, est, on l'a vu, de ne pas s'être suffisamment intéressée aux variations génériques ou intermédiatiques. L'étude de l'adaptation des figures du cycle de Guèvremont montre à quel point les divers changements de genre bouleversent l'écriture et comment le passage d'une rhétorique du roman aux rhétoriques du radioroman et du téléroman est complexe et souvent difficile.

\section{Bibliographie}

\section{OEuvres de Germaine Guèvremont}

«L'arrivée de Venant » et "L'abandon », Gants du ciel, no 2, décembre 1943, p. 21-32 (première version des chapitres I et XVII du Survenant).

Le Survenant, édition critique par Yvan G. Lepage, Montréal, Presses de l'Université de Montréal, coll. «Bibliothèque du Nouveau Monde », [1945] 1989. 
Marie-Didace, édition critique par Yvan G. Lepage, Montréal, Presses de l'Université de Montréal, coll. « Bibliothèque du Nouveau Monde », [1947] 1996.

Le Survenant, Radioroman (CBF, 31 août 1953 - 6 mai 1955; repris à CKVL, septembre 1962 - juin 1965), Bibliothèque et archives Canada, Fonds Germaine Guèvremont, LMS 0260, 2004-03, série 1.

Le Survenant, téléroman, saisons 1 à $3, \mathrm{CBFT}$, réalisation $\mathrm{M}$. Leroux, BanQ, Fonds de l'Institut de recherches sur la littérature radiophonique et télévisuelle, (1 mai 1956 - 9 juillet 1957); Fonds Germaine Guèvremont, LMS 0260, 200403, série 2 [Première saison : 2 novembre 1954 - 26 avril 1955, 22 épisodes. Deuxième saison : 18 octobre 1955 - 10 juillet 1956, 21 épisodes. Troisième saison : 16 octobre 1956 - 9 juillet 1957, 39 épisodes.]

\section{Autres œuvres citées}

Aubry Danielle, Du roman-feuilleton à la série télévisuelle. Pour une rhétorique du genre et de la sérialité, Berne, Peter Lang, 2006.

Besson Anne, D'Asimov à Tolkien. Cycles et séries dans la littérature de genre, Paris, CNRS éditions, 2004.

BACRY Pierre, Les figures de style, Paris, Belin, coll. "Sujets », 1992.

DÉCARIE David, «Le relais des survenants chez G. Guèvremont », Voix et images, vol. XXVI, n 2, hiver 2001, p. 359-383.

DÉCARIE David, «Résonances. Interfiguralité chez G. Guèvremont», dans La rhétorique au féminin (sous la direction d'Annette Hayward), Québec, Nota bene, 2006, p. 315-333. 
DUPRIEZ Bernard, Gradus. Les procédés littéraires, Paris, Union générale d'Éditions, coll. 10/18, 1984.

Ducrot Oswald, «Esquisse d'une théorie polyphonique de l'énonciation », Le dire et le dit, Paris, Minuit, 1984.

Genette Gérard, Figures III, Paris, Seuil, 1972.

GenetTe Gérard, Métalepse, Paris, Seuil, 2004.

PELlETIER-Dlamini Louis, "Germaine Guèvremont. Rencontre avec l'auteur du Survenant », Châtelaine, vol. VIII, no 4 , avril 1967, p. 32, 33, 84, 86, 88.

HAMON Philippe, «Pour un statut sémiologique du personnage ", Gérard Genette, Tzvetan Todorov, Roland Barthes, Wolfgang Johannes Kayser, Wayne C. Booth, Philippe Hamon, Poétique du récit, Paris, Seuil, coll. «Points », 1977.

RABATEl Alain, $\mathrm{La}$ construction textuelle $d u$ point de vue, Lausanne, Delachaux et Niestlé, 1998.

SMART Patricia, Écrire dans la maison du père, Montréal, Québec/Amérique, 1988.

TRAVERSo Véronique, L'analyse des conversations, Paris, Nathan, coll. « $128 », 1999$.

White Roger M., The Structure of Metaphor, Cambridge, Blackwell Publishers, 1996.

\section{Sites Internet}

KAEMPFER Jean et Filippo ZANGHI (2003). La voix narrative, Méthodes et problèmes, Genève, Département de français moderne http://www.unige.ch/lettres/framo/enseignements/methodes/ vnarrative/

JENNY, Laurent (2003). Les figures de rhétorique, Méthodes et problèmes, Genève, Département de français moderne, 
http://www.unige.ch/lettres/framo/enseignements/metho des/frhetorique/

Jenny, Laurent (2003). Dialogisme et polyphonie, Méthodes et problèmes, Genève, Département de français moderne http://www.unige.ch/lettres/framo/enseignements/metho des/dialogisme/

\title{
Résumé
}

Le Cycle du Survenant est une œuvre plurigénérique et intermédiale. Les divers changements de genre qui traversent l'œuvre provoquent de véritables bouleversements dans l'écriture de Guèvremont, notamment au niveau des figures de style. L'étude du passage des figures des trois premiers chapitres du roman Le Survenant vers le radioroman et le téléroman du même nom permet de mieux comprendre les spécificités rhétoriques de ces trois genres ainsi que les défis posés par les adaptations radiophonique et télévisuelle.

\begin{abstract}
The Survenant cycle is multi-genre and intermedial. The many shifts in genre that took place over the course of Guèvremont's career led to important changes in her writing, notably in terms of the rhetorical figures used. The study of the transformation of figures from the first three chapters of Le Survenant in the radio and televisual adaptations of this novel provides a better understanding of the rhetorical singularities of these three genres, as well as of the challenges posed by adaptations to different media.
\end{abstract}

